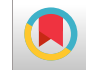

\title{
Approach to Growing Pains in Childhood in a Familial Mediterranean Fever Endemic Region
}

\author{
Ilke Beyitler,', ${ }^{1}$ and Salih Kavukcu' ${ }^{1}$ \\ ${ }^{1}$ Department of Pediatrics, Division of Pediatric Nephrology and Rheumatology, Near East University Hospital, Nicosia, Cyprus \\ "Corresponding author: Ilke Beyitler, Near East University Hospital, Department of Pediatrics, 99138, Nicosia, Cyprus. Tel: +90-3924440535, E-mail: ilkebeyit82@yahoo.com
}

Received 2017 March 27; Revised 2017 June 12; Accepted 2017 September 02.

\begin{abstract}
Growing pain, still preserving its charm, is the most common form of recurrent musculoskeletal pain in childhood. Approach to diagnosis requires significant care in geographical regions with familial Mediterranean fever(FMF) endemicity. We discuss data and a current approach to growing pains in children in an FMF endemic region. The symptomatology may be confused with different diseases. Although unnecessary tests may be performed to reach diagnosis, sometimes patients may be misdiagnosed as having growing pain without revealing the primary underlying pathology. Definite algorithms are not developed for diagnosis of growing pains. Diagnosis is mainly based on typical clinical features of the condition. However laboratory tests and imaging methods may be used for diagnosis and differential diagnosis especially in atypical course suggesting diseases such as inflammatory ones or malignancies. Vitamin D deficiency should be investigated to give supplementation if necessary. Some helpful algorithms may be used in evaluation of patients. In order to evaluate myalgia as growing pain in areas where FMF is endemic, fever, abdominal pain, elevated acute phase reactants and history of FMF and/or amyloidosis in family should be carefully excluded. It is important to inform parents about the alarming symptoms of extremity pain and when to urge medical advice again.
\end{abstract}

Keywords: Growing Pain, Fever, Children, Diagnosis, Management

\section{Introduction}

Growing pains, first described in 1823 by Duchamp, are the most common form of recurrent musculoskeletal pain in childhood affecting especially the muscles of lower extremities. Anterior thigh, calf and posterior of knee are the main areas that ache. Pain is generally bilateral and intermittent but not necessarily with a regular pattern, occurs in the evening or at night, may or may not awaken the child and resolves spontaneously or by rubbing the legs. However analgesics may sometimes be required $(1,2)$. Positive family history for growing pain is observed. Duration of pain differs from minutes to hours that may be mild or severe in intensity. There are pain free intervals ranging between days to months. Children aged 3 - 14 years are defined to be affected showing a peak at 6 years. In some rare reports growing pains were indicated to persist after puberty. Prevalence of growing pains is found to have a range of $2.6 \%-49.4 \%$ in different study results. Physical examination and laboratory tests of these children are normal which are useful to exclude other conditions such as arthritis and bone tumors $(3,4)$.

\section{Etiopathogenesis}

Although considered to be due to rapid growth of bones and later as part of rheumatic fever in previous centuries, growing pain has been shown not to be related with these conditions as growth is fastest in pubertal period and rheumatic fever is an inflammatory disorder. However the name "growing" has persisted until today $(1,5)$.

\subsection{Anatomical Reasons}

Anatomical disorders such as flat feet, scoliosis, lordosis, genu valgum had been considered as causing growing pains because they lead to posture defects. Mechanical instability of flat feet with hindfoot valgus is reported as a cause of growing pain in children. Shoe inserts were shown to reduce growing pain intensity and frequency in a controlled trial. However later studies showed that foot posture and growing pains are not related. Obesity was also related with growing pain but a later study found no correlation of growing pains with body weight. Therefore the anatomical theory could not be verified with strong evidence $(6,7)$.

Generalized joint hypermobility and knee hypermobility were shown in a cross - sectional study to be associated 
with growing pains in children. Hypermobility is the increased range of motion of joints that is assessed with an $\geq$ 5.9 score on Beighton scale. Pain of the hypermobile joints increase after exercise and relieve with rest. Pain may be explained with hypermobility as sometimes growing pains ocur after increased physical activity $(8,9)$.

\subsection{Decreased Pain Threshold}

Children with growing pains were demostrated to have more tender points and lower pain thresholds than control groups measured by dolorimeter strongly suggesting that it may be a non-inflammatory pain syndrome (10). After 5 years the same cohort were examined again and $49 \%$ had persisting growing pains with less severe and less frequent episodes, while $51 \%$ had complete pain resolution. Persistent group were again found to have lower pain thresholds on anterior tibia and fibromyalgia points. Which also shows that growing pain may be a pain amplification syndrome (11). Somatosensory test responses to cold, vibration and deep pressure were also found to be increased in children with growing pains (12).

\subsection{Psychosocial Factors}

Emotional stress has been shown to be more common in children that have growing pain. Psychological distress of the family and children increase the development of pain syndromes involving not only limb pain, but also headache and abdominal pain. Mothers of children who have growing pain were found to have increased depression levels. In a small study group, quality of life scores in children with growing pain were found to be less than those in children without it $(13,14)$.

\subsection{Bone Strength and Fatigue}

As growing pains occur in evening or at night and more after increased physical activity, these also suggest that growing pain may be an overuse syndrome (15). Quantitative ultrasound of bones gives measures correlated with dual energy x-ray absorptiometry results. Bone speed of sound was measured by quantitative ultrasound and was decreased in radius and especially in tibia of children that have growing pain (16).

\subsection{Family History}

Some of the growing pain cases have a positive family history of growing pain suggesting a genetic association. A parent or a sibling that experienced growing pain was reported in 70\% of growing pain patients (13). A 3 - 16 years aged twin family study added that growing pains are not only familial but also a genetic influence is shown in twins. Monozygotic twins were in more concordance with growing pains than dizygotic twins as well (17).

\subsection{Vitamin D deficiency}

Vitamin D deficiency and insufficiency were found to have a high prevalence concerning children with growing pain. These children mostly had pain during winter and autumn and the time of decrease in 25-hydroxyvitamin D [25 (OH) D] levels were compatible with that seasonal variation (18).

In a prospective cohort study including 120 children that had growing pain and pain was measured with visual pain scale, $86.6 \%$ were shown to have vitamin D deficiency. After oral vitamin supplementation, $25(\mathrm{OH})$ D levels increased and pain scores decreased significantly (19). A similar study with 33 children having growing pain, demonstrated a $93.9 \%$ of $25(\mathrm{OH})$ D deficiency and insufficiency and $73.1 \%$ of low bone mineral status measured by quantitative ultrasound. Three months after vitamin D treatment, $25(\mathrm{OH})$ D levels increased and pain severity evaluated with a questionnare decreased. Twenty four months later, pain intensity decreased and bone mineral status improved (20). Hundred children aged 5 - 12 years who had growing pain were investigated and $95 \%$ were found to have vitamin D insufficiency, 72 \% of whom had vitamin D deficiency. It is suggested that growing pain is an initial manifestation of bone matrix alterations in children (21).

Growing pain is not an expected entity in first year of life when growth is fastest and this can be explained with routine vitamin D supplementation in that period. It can also be speculated that growing pain may not be considered in infancy due to difficulty in diagnsosis.

\subsection{Restless Leg Syndrome}

Restless leg syndrome (RLS) is a condition in which there is leg pain that is worse at night, feeling urge to move legs and pain worsening with inactivity. Although not clear, a dopaminergic mechanism may be responsible in etiology. It was reported that a group of children with growing pain have been found to meet criteria of RLS. They also have a family history of RLS and symptoms were more severe requiring use of analgesics (22). In a case control study concerning pregnant women with RLS, they had a higher history of growing pain in childhood compared to the control group strengthening that growing pains are a predictor of RLS (23).

\subsection{Blood Perfusion Status}

Bone scans with scintigraphy of children with growing pain were not different form the control group in a study unlike migraine attacks in which vascular perfusion is altered (24). 


\subsection{Trace Elements}

Hair of children with growing pains was investigated and levels of lead and zinc were increased while those of copper and magnesium decreased. However these findings could not be validated for use (13).

Etiopathogenesis of growing pains and an approach schema are given in Figures 1 and 2 respectively.

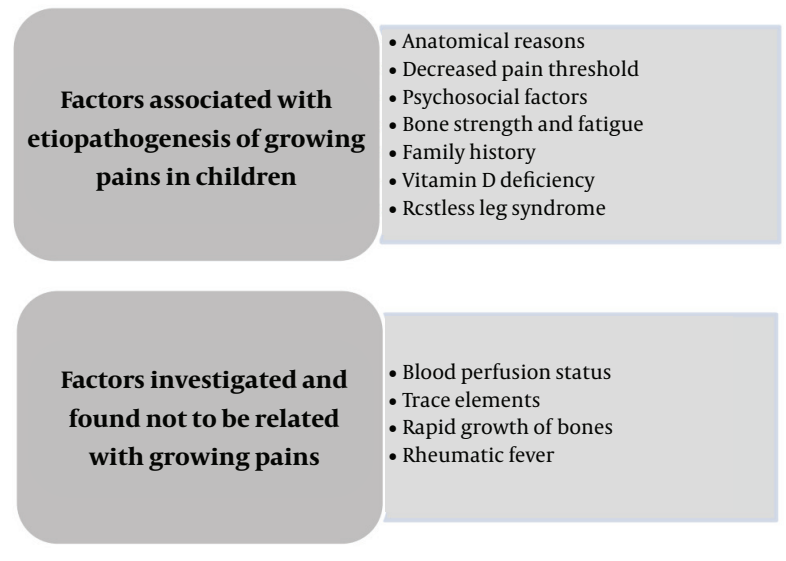

Figure 1. Etiopathogenesis of Growing Pains

\section{What Should Be Done?}

Diagnosis of growing pain is based on typical features of the condition and in fact there is routinely no need to do any laboratory tests. Complete blood count (CBC), erythrocyte sedimentation rate (ESR), calcium, phosphorus, rheumatoid factor results and radiographs are normal in growing pain. Alkaline phosphatase levels may be high due to growth in childhood. Atypical course, systemic symptoms or signs of inflammation suggest other diseases that should be excluded with laboratory tests and/or imaging methods (25). Ethnicity should also be regarded.

Recently, an easy questionnaire including nine questions were given to parents of children aged 4 - 7 years having musculoskeletal pain. The questions were about pain localization, intensity and distribution during the day. A score of $\geq 8$ was regarded for growing pain diagnosis and a score $<6$ excluded diagnosis. Diagnosis of growing pain was compatible with the diagnosis of orthopedics department in nearly all cases and the scale was suggested to be a reliable screening tool (26).

\section{Diseases That Should Be Excluded}

There are various causes of extremity pain in children. Major groups are rheumatological diseases, infec- tions, malignancies, trauma, noninflammatory pain syndromes, congenital or mechanical abnormalities, developmental bone diseases and vascular related causes. A detailed history and complete physical examination are the key points in evaluating these cases that provide enough clues with typical features of the condition. However, in case of fever, weight loss, pain continuing the next morning or morning stiffness, limping, signs of joint inflammation or persisting asymmetric pain, the patient should be checked with CBC, ESR, biochemical tests and if necessary, bone imaging as well $(1,25,27)$.

Familial mediterranean fever (FMF) is an autosomal recessively inherited disorder with recurrent fever and polyserositis attacks that may cause renal failure due to amyloidosis when it is untreated. Elevation of acute phase reactants are observed during attack periods of FMF. Although the majority of attacks are prominent with fever, peritonitis, pleuritis and arthritis, some cases are reported to have only myalgia as a presenting symptom and were diagnosed to have homozygous mutations on the MEFV gene. It is important to distinguish between recurrent myalgia due to FMF and growing pains especially in children living in the Mediterranean region or those of Turk, Armenian, Arab or Jew ethnicity (28-30) In order to evaluate myalgia as growing pain in areas where FMF is endemic, fever, abdominal pain, elevated acute phase reactants and history of FMF and/or amyloidosis in family should be carefully excluded.

Tumor necrosis factor receptor associated-periodic syndrome (TRAPS) is presented with 1 - 4 week attacks of recurrent fever, myalgia, serositis, arthritis, ocular involvement and skin lesions such as macular rash. Attacks may be triggered by infections, trauma or exercise. Although less common than FMF, TRAPS patients also have the risk for amyloidosis. Inflammation markers are positive and genetical diagnosis of TNFRSF1A mutations are possible (31).

Juvenile idiopathic arthritis (JIA) patients may have myalgia but they generally present with fatigue, arthritis, fever, abdominal pain, chest pain and weight loss. In a multicentre study with 136 children diagnosed as JIA, the most common clinical presentations were fever, arthritis, rash, serositis, lymphadenopathy and hepatosplenomegaly. Diagnosis is made by clinical features and typical laboratory findings with elevated acute phase reactants. JIA is a disease to be excluded in children complaining of recurrent extremity pain (32).

Systemic lupus erythematosus (SLE) patients often have myalgia and arthralgia with fever, rashes, alopecia, photosensitivity, nephritis, neuropsychiatric disease, serositis and hematological involvement. Myalgia and arthralgia that especially involves small joints of the hands, resolves after SLE diagnosis and appropriate treat- 


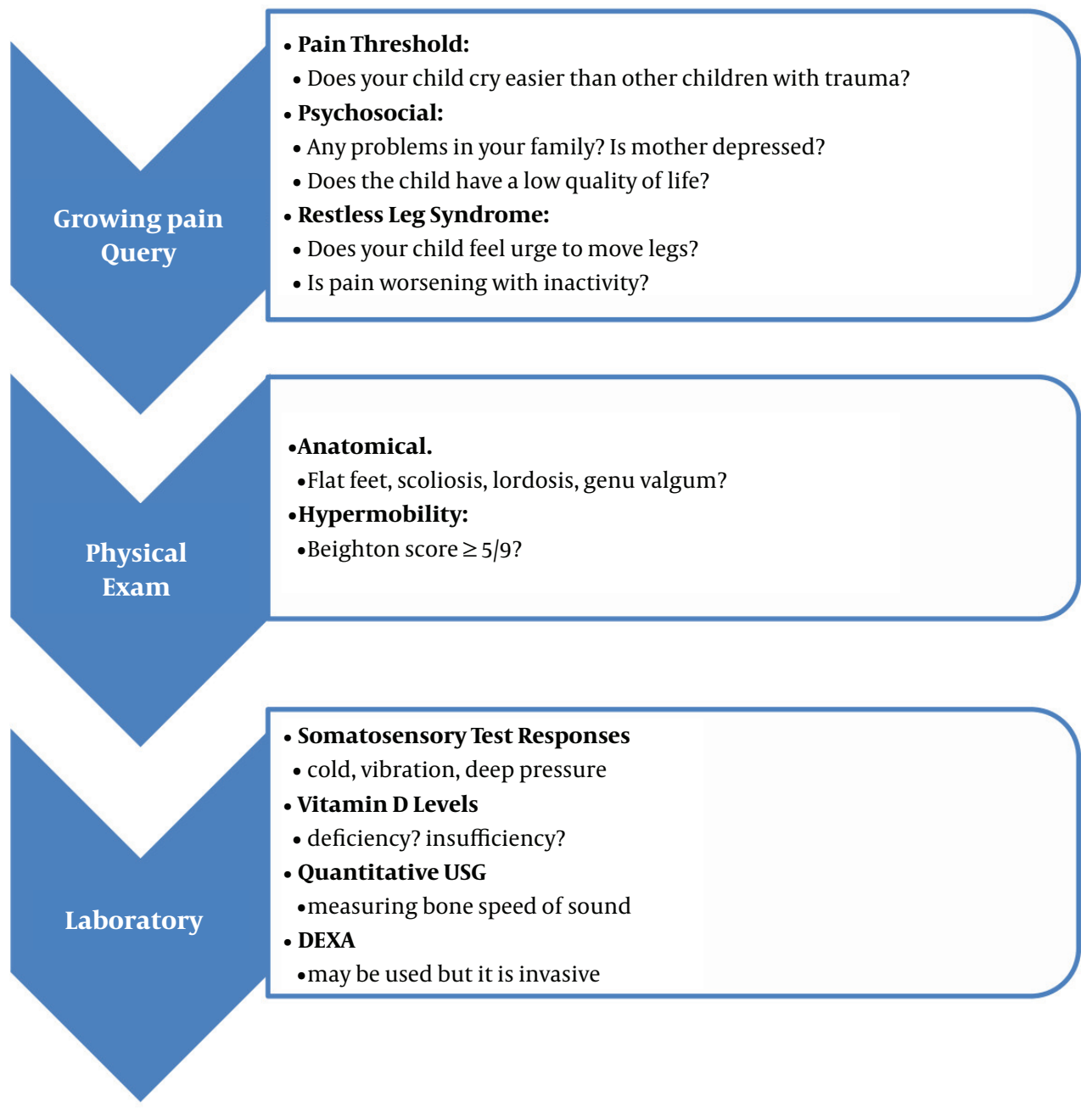

Figure 2. Approach to Growing Pains in Childhood

ment (33).

Macrophagic myofasciitis in children is thought to be due to adjuvants in vaccines presenting with myalgia, arthralgia, fatigue, elevated ESR and creatine kinase. The patients may have hypotonia, developmental delay and failure to thrive. Muscle biopsy shows macrophage and CD8+ T lymphocyte infiltration, in which aluminium hydroxide in macrophages are seen on electron microscopy (34).

In juvenile polymyositis, children may have myalgia, as well as falling attacks due to distal weakness, Raynaud phenomenon, weight loss, lung disease and cardiac anomalies. Diagnosis is possible with muscle biopsy (35).
Juvenile Sjøgren syndrome that involves chronic inflammation of exocrine glands, may present with myalgia and patients may have features like fatigue, low grade fever, arthralgia, ocular symptoms, dry mouth, difficulty in swallowing and parotitis. Autoantibodies ANA, anti-Ro, anti-La are checked for diagnosis (36).

In vasculitis syndromes myalgia may occur as well as a broad spectrum of signs such as arthralgia, arthritis, myositis, fever, weight loss, fatigue, skin lesions, neurological lesions, serositis, hypertension, hematuria, renal failure, pulmonary and cardiac involvement, increased acute phase reactants and autoantibodies with leukocytosis, anemia or thrombocytosis (37). 
One of the vasculitis syndromes, sytemic polyarteritis nodosa (PAN) is characterised by myalgia, muscle tenderness and patients may also have malaise, fever, weight loss, skin involvement, arthritis, abdominal pain, cardiac ischemia, renal features like hematuria, proteinuria, hypertension and neurological manifestations. In populations with FMF endemicity, genetic susceptibility to develop PAN has been suggested to be increased, so PAN should also be considered in differential diagnosis of growing pains (38).

Reactive arthritis causes joint and extraarticular features such as myalgia and erythema on affected area after certain infections. It generally affects the lower limbs, is asymmetric, oligoarticular with a history or laboratory evidence of a preceeding infection (39).

A 15 year old female patient had bilateral knee pain occuring every winter for 5 years. She had hypermobility of joints, acute phase reactants were negative and the patient was diagnosed as growing pain for 5 years in different medical centres. However, the continuity of pain until puberty, occurence of pain during daytime besides nights and pain being on the knee joints suggested another diagnosis. Bilateral knee MRI revealed chondromalacia patellae that is degradation of patellar cartilage and the patient was recommended analgesics and modification of activity (40).

A seven year old boy had recurrent leg and knee pain attacks after physical activity. Laboratory tests and leg imaging were normal leading to a misdiagnosis of growing pain. However some attacks were associated with swelling of knee joint and pain occured both at night and day. The patient was finally diagnosed as intermittent overuse injury on the opposite side to an enlarged ischiopubic synchondrosis (41).

\section{How to Manage?}

The key point in growing pain is that the clinician should be sure of this diagnosis. We can turn to this diagnosis after a thorough differential diagnosis. Otherwise a malignancy, an infection or a rheumatologic problem may be misdiagnosed leading to a very dangerous time loss.

A 5 year old girl with severe arm and leg pain at night twice a week had normal pyhsical examination, normal laboratory investigation and normal MRI of elbows. FMF mutation was also normal. Each time pain disappeared the next morning but the mother was afraid and anxious demanding blood tests frequently. After evaluation and follow up at child psychiatry department, the child and mother were more confident and attacks decreased dramatically.

A recent summary of randomized trials regarding cognitive behavioral therapy for children with fibromyalgia concluded that psychosocial therapy is not definitely effective for pain relief because post-treatment change scores are not available (42). In regard to growing pains, it is important to reassure both the parents and the child that growing pains are benign and resolve with time. Typical features are enough for this diagnosis. Behavioral support is effective to decrease pain and fear (43). Children with growing pains were questioned for sleep characteristics and found to have increased sleep terror and disturbed patterns such as walking while asleep suggesting an association between parasomnia and growing pains (44). These findings also support the need for psychological interventions.

Application of warmth and massage over the affected area may be tried for pain relief. The pain may resolve because of being a self limiting condition or because the parents are paying attention to the child by these local techniques (45). An exercise program involving the streching of quadriceps, hamstrings and gastrosoleus muscles were shown to decrease the pain episodes and parents may be encouraged to do so daily at home. Physical therapy will also improve motivation, fitness and psychological status simultaneously (46).

Shoe inserts were effective for children with pronated foot posture in a small sample controlled study. Therefore these inserts may be used only in selected cases with postural defects (47).

Chiropractic spinal manipulative therapy was reported to be effective for resolution of frequent growing pains in a case series having spinal segmental tenderness and hypertonicity of muscles on physical examination. This therapy was defined as high velocity, low amplitude thrusts on segments of spinal dysfunction. However further researches are needed in that area (48).

Analgesics may be used if necessary, paracetamol or ibuprofen can be a good choice. Naproxen as a long acting analgesic may be useful in case of severe pain, interruption of activity or sleep $(1,10)$.

As bone strength has a role in etiopathogenesis of growing pains, vitamin D levels are valuable especially when pain occurs in winter seasons. Supplementation of vitamin $\mathrm{D}$, if necessary, was shown to decrease pain effectively and improve bone health $(20,22)$.

\section{Conclusion}

Growing pain is a common form of noninflammatory pain syndrome that resolves with time but sometimes may persist after puberty. In these cases, pain threshold is low on typical regions and fibromyalgia points suggesting that it is a pain amplification syndrome. Typical features are enough for diagnosis but it is important to check vitamin 
D levels of these children for supplementation if necessary and advising them to have more outdoor activities in order to benefit from sunshine adequately. FMF should firstly be excluded in order to interpret myalgia as growing pain in a FMF endemic region.

Parents should be informed about the alarming symptoms of extremity pain and when to urge medical advise again.

\section{References}

1. Lowe RM, Hashkes PJ. Growing pains: a noninflammatory pain syndrome of early childhood. Nat Clin Pract Rheumatol. 2008;4(10):542-9. doi:10.1038/ncprheum0903. [PubMed: 18762787].

2. Uziel Y, Hashkes PJ. Growing pains in children. Pediatr Rheumatol Online J. 2007;5:5. doi: 10.1186/1546-0096-5-5. [PubMed: 17550631].

3. Evans AM. Growing pains: contemporary knowledge and recommended practice. J Foot Ankle Res. 2008;1(1):4. doi:10.1186/1757-1146-1-4. [PubMed: 18822152].

4. Pavone V, Lionetti E, Gargano V, Evola FR, Costarella L, Sessa G. Growing pains: a study of 30 cases and a review of the literature.JPediatr Orthop. 2011;31(5):606-9. doi: 10.1097/BPO.0b013e318220ba5e. [PubMed: 21654473].

5. Mohanta MP. Growing pains: practitioners' dilemma. Indian Pediatr. 2014;51(5):379-83. [PubMed: 24953579].

6. Evans AM, Scutter SD. Are foot posture and functional health different in children with growing pains? Pediatr Int. 2007;49(6):991-6. doi: 10.1111/j.1442-200X.2007.02493.x. [PubMed: 18045309].

7. Kaspiris A, Zafiropoulou C. Growing pains in children: epidemiological analysis in a Mediterranean population. Joint Bone Spine. 2009;76(5):486-90. doi: 10.1016/j.jbspin.2009.09.001. [PubMed: 19793673].

8. Viswanathan V, Khubchandani RP. Joint hypermobility and growing pains in school children. Pediatr Rheumatol. 2008;26:962-6.

9. Sperotto F, Balzarin M, Parolin M, Monteforte N, Vittadello F, Zulian F. Joint hypermobility, growing pain and obesity are mutually exclusive as causes of musculoskeletal pain in schoolchildren. Clin Exp Rheumatol. 2014;32(1):131-6. [PubMed: 24093536].

10. Hashkes PJ, Friedland O, Jaber L, Cohen HA, Wolach B, Uziel Y. Decreased pain threshold in children with growing pains.J Rheumatol. 2004;31(3):610-3. [PubMed: 14994414].

11. Uziel Y, Chapnick G, Jaber L, Nemet D, Hashkes PJ. Five-year outcome of children with "growing pains": correlations with pain threshold.J Pediatr. 2010;156(5):838-40. doi: 10.1016/j.jpeds.2009.11.078. [PubMed: 20171654].

12. Pathirana S, Champion D, Jaaniste T, Yee A, Chapman C. Somatosensory test responses in children with growing pains. J Pain Res. 2011;4:393-400. doi: 10.2147/JPR.S24875. [PubMed: 22247622].

13. Evans A, Scutter S, Lang L, Dansie B. Growing pains in young children: a study of the profile, experiences and quality of life issues of four to six year old children with recurrent leg pain. Foot. 2006;16:120-4.

14. Oberklaid F, Amos D, Liu C, Jarman F, Sanson A, Prior M. "Growing pains": clinical and behavioral correlates in a community sample.J Dev Behav Pediatr. 1997;18(2):102-6. [PubMed: 9113591].

15. Uziel Y, Chapnick G, Oren-Ziv A, Jaber L, Nemet D, Hashkes PJ. Bone strength in children with growing pains: long-term follow-up. Clin Exp Rheumatol. 2012;30(1):137-40. [PubMed: 22325064].

16. Friedland O, Hashkes PJ, Jaber L, Cohen HA, Eliakim A, Wolach B, et al. Decreased bone speed of sound in children with growing pains measured by quantitative ultrasound. J Rheumatol. 2005;32(7):13547. [PubMed: 15996077].
17. Champion D, Pathirana S, Flynn C. Growing pains: Twin family study evidence for genetic susceptibility and a genetic relationship with restless legs syndrome. EurJ Pain. 2012.

18. Park MJ, Lee J, Lee JK, Joo SY. Prevalence of Vitamin D Deficiency in Korean Children Presenting with Nonspecific Lower-Extremity Pain. Yonsei Med J. 2015;56(5):1384-8. doi: 10.3349/ymj.2015.56.5.1384. [PubMed: 26256984].

19. Vehapoglu A, Turel O, Turkmen S, Inal BB, Aksoy T, Ozgurhan G, et al. Are Growing Pains Related to Vitamin D Deficiency? Efficacy of Vitamin D Therapy for Resolution of Symptoms. Med Princ Pract. 2015;24(4):332-8. doi: 10.1159/000431035. [PubMed: 26022378].

20. Morandi G, Maines E, Piona C, Monti E, Sandri M, Gaudino R, et al Significant association among growing pains, vitamin D supplementation, and bone mineral status: results from a pilot cohort study. J Bone Miner Metab. 2015;33(2):201-6. doi: 10.1007/s00774-014-0579-5. [PubMed: 24633492].

21. Qamar S, Akbani S, Shamim S, Khan G. Vitamin D levels in children with growing pains. J Coll Physicians Surg Pak. 2011;21(5):284-7. [PubMed: 21575536].

22. Rajaram SS, Walters AS, England SJ, Mehta D, Nizam F. Some children with growing pains may actually have restless legs syndrome. Sleep. 2004;27(4):767-73. [PubMed: 15283013].

23. Balendran J, Champion D, Jaaniste T, Welsh A. A common sleep disorder in pregnancy: restless legs syndrome and its predictors. Aust $N Z$ J Obstet Gynaecol. 2011;51(3):262-4. doi: 10.1111/j.1479-828X.2011.01294.x. [PubMed: 21631448].

24. Hashkes PJ, Gorenberg M, Oren V, Friedland O, Uziel Y. "Growing pains" in children are not associated with changes in vascular perfusion patterns in painful regions. Clin Rheumatol. 2005;24(4):342-5. doi: 10.1007/s10067-004-1029-x. [PubMed: 16034648].

25. Asadi-Pooya AA, Bordbar MR. Are laboratory tests necessary in making the diagnosis of limb pains typical for growing pains in children? Pediatr Int. 2007;49(6):833-5. doi: 10.1111/j.1442-200X.2007.02447.x. [PubMed: 18045281].

26. Vasilopoulou M, Spathis A, Myriokefalitakis N, Zaferopoulou F, Paspati I, Tsolia M. Development of a screening tool for children's growing pains: validation, reliability control and clinical evaluation. TurkJ Pediatr. 2015;57(5):467-74. [PubMed: 27411413].

27. de Inocencio J. Musculoskeletal pain in primary pediatric care: analysis of 1000 consecutive general pediatric clinic visits. Pediatrics. 1998;102(6):E63. [PubMed: 9832591].

28. Kavukcu S, Turkmen MA, Soylu A, Bayram MT, Ulgenalp A. Myalgia as a symptom of familial Mediterranean fever in children. Rheumatol Int. 2012;32(11):3705-6. doi: 10.1007/s00296-011-2167-5. [PubMed: 22020390].

29. Kavukcu S, Turkmen M, Soylu A, Kasap B, Tatli Gunes B. Skin and muscle involvement as presenting symptoms in four children with familial Mediterranean fever. Clin Rheumatol. 2009;28(7):857-60. doi: 10.1007/s10067-009-1138-7. [PubMed: 19240979].

30. Soylu A, Kasap B, Turkmen M, Saylam GS, Kavukcu S. Febrile myalgia syndrome in familial Mediterranean fever. J Clin Rheumatol. 2006;12(2):93-6. doi: 10.1097/01.rhu.0000208635.64537.10. [PubMed: 16601545].

31. Radhakrishna SM, Grimm A, Broderick L. Novel mutation identified in severe early-onset tumor necrosis factor receptor-associated periodic syndrome: a case report. BMC Pediatr. 2017;17(1):108. doi: 10.1186/s12887-017-0856-2. [PubMed: 28427379].

32. Behrens EM, Beukelman T, Gallo L, Spangler J, Rosenkranz M, Arkachaisri T, et al. Evaluation of the presentation of systemic onset juvenile rheumatoid arthritis: data from the Pennsylvania Systemic Onset Juvenile Arthritis Registry (PASOJAR). J Rheumatol. 2008;35(2):343-8. [PubMed: 18085728].

33. Kumar S, Nair S, Rajam L. Case series of pediatric systemic lupus erythematosus from Kerala: comparison with other Indian series. Int J Rheum Dis. 2010;13(4):391-5. doi: 10.1111/j.1756-185X.2010.01536.x. [PubMed: 21199476]. 
34. Gherardi RK, Authier FJ. Macrophagic myofasciitis: characterization and pathophysiology. Lupus. 2012;21(2):184-9. doi: 10.1177/0961203311429557. [PubMed: 22235051].

35. Shah M, Mamyrova G, Targoff IN, Huber AM, Malley JD, Rice MM, et al. The clinical phenotypes of the juvenile idiopathic inflammatory myopathies. Medicine (Baltimore). 2013;92(1):25-41. doi: 10.1097/MD.ob013e31827f264d. [PubMed: 23263716].

36. Venables PJ. Management of patients presenting with Sjogren's syndrome. Best Pract Res Clin Rheumatol. 2006;20(4):791-807. doi: 10.1016/j.berh.2006.05.003. [PubMed: 16979538].

37. Jennette JC. Overview of the 2012 revised International Chapel Hill Consensus Conference nomenclature of vasculitides. Clin Exp Nephrol. 2013;17(5):603-6. doi: 10.1007/s10157-013-0869-6. [PubMed: 24072416].

38. Yalcinkaya F, Ozcakar ZB, Kasapcopur O, Ozturk A, Akar N, Bakkaloglu A, et al. Prevalence of the MEFV gene mutations in childhood polyarteritis nodosa. J Pediatr. 2007;151(6):675-8. doi 10.1016/j.jpeds.2007.04.062. [PubMed: 18035151].

39. Naranje S, Kelly DM, Sawyer JR. A Systematic Approach to the Evaluation of a Limping Child. Am Fam Physician. 2015;92(10):908-16 [PubMed: 26554284].

40. Beyitler I, Kocaoglu M, Kavukcu S. Bilateral knee pain in an adolescent girl; A case of chondromalacia patella. Turk Clin J Pediatr.
41. Beyitler I, Kavukcu S. A case of Van-neck Odelberg disease and intermittent overuse injury. Arch Rheumatol. 2016;31(4):381-3.

42. Cohen EM, Fletcher AM, Darshan HM, Lee YC. A systematic review of psychosocial therapies for children with rheumatic diseases. Ped Rheumatol. 2017;15(6).

43. Goodyear SF, Arrol B. Growing pains, parents and children need reassuring about this self-limiting condition of unkown cause. BMJ. 2006;333:456-7.

44. Aeschlimann FA, Werner H, Jenni OG, Saurenmann RK. Are growing pains a parasomnia. Ped Rheumatol. 2012;10(Supp 1):A78. .

45. Weiser P. Approach to the patient with noninflammatory musculoskeletal pain. Pediatr Clin North Am. 2012;59(2):471-92. doi: 10.1016/j.pcl.2012.03.012. [PubMed: 22560580].

46. Baxter MP, Dulberg C. "Growing pains" in childhood-a proposal for treatment. J Pediatr Orthop. 1988;8(4):402-6. [PubMed:3292578].

47. Evans AM. Relationship between growing pain and foot posture in children: single case experimental design in clinical practice. J Am Pediatr Med Assoc. 2003;93:111-7.

48. Alcantara J, Davis J. The chiropractic care of children with "growing pains": a case series and systematic review of the literature. Complement Ther Clin Pract. 2011;17(1):28-32. doi: 10.1016/j.ctcp.2010.08.006. [PubMed: 21168111]. 